\title{
Structure and Viscoelastic Properties of Poly(Ether-Block-Amide) Thermoplastic Elastomers with No Ester Linkages
}

\author{
Shuichi MaEdA ${ }^{*} \dagger$ and Hiroshi OKushita ${ }^{* *}$ \\ " Advanced Science and Innovational Research Center, Organization for Research Initiatives, Yamaguchi University, \\ 2-16-1, Tokiwadai, Ube, Yamaguchi 755-8611, Japan \\ ** Practical Engineering Education Program, Academic Foundations Programs, Kanazawa Institute of Technology, \\ 7-1 Ohgigaoka, Nonoichi, Ishikawa 921-8501, Japan \\ (Received : November 22, 2018)
}

\begin{abstract}
We investigated the structure and viscoelastic properties of a series of poly(ether-block-amide) thermoplastic elastomers consisting of hard polyamide segments and soft polyether segments with no ester linkages. All thermoplastic elastomers used in this study exhibited heterogenous morphologies with crystalline lamella in the solid state. The heterogeneities and solubilities in the amorphous regions of the elastomers depended on the sample composition. We did not observe a clear, slow relaxation mechanism characteristic of phase-separated liquids for the dynamic moduli of samples in the molten state. This result shows that the melted samples had a homogeneous structure due to the good miscibility between the polyamide and polyether segments.
\end{abstract}

Key Words: Poly(ether-block-amide) thermoplastic elastomer / Viscoelastic properties / Structure in solid state / Glass transition temperature / Structure in molten state

\section{INTRODUCTION}

A poly(ether-block-amide) thermoplastic elastomer (PEA) has a microphase-separated structure in which the hard segments (HSs) and soft segments (SSs) form domains. The mechanical properties of PEA depend strongly on the phase-separated structure ${ }^{1-6)}$. A unique property of PEA is its thermal adhesion to different kinds of polymers, such as polyurethane and crosslinked rubber ${ }^{5-7)}$. However, there are many unsolved issues around the thermal adhesive properties of PEA with other polymers. Because the thermal adhesion between different polymers is characteristic of the two polymers in contact with each other-which are heated over their glass transition temperatures $\left(T_{\mathrm{g}} \mathrm{s}\right)$ and melting temperatures, cooled, and solidified - it is important to clarify the structures and viscoelastic properties of PEA in solid and molten states.

We prepared a series of PEAs with different compositions composed of 12-aminododecanoic acid (ADA) and adipic acid (AA) as the HS and polyether as the SS. The chemical structure of PEA is shown in Fig. 1. The PEAs used in this study are multiblock copolymers composed of HSs and SSs that contain no ester linkages, thus conferring

\footnotetext{
$\dagger$ Corresponding author.

E-mail : maeshu1@yamaguchi-u.ac.jp

Tel: +81-836-85-9944; Fax: +81-836-85-9962
}

excellent fluidity and resistance to hydrolysis compared with PEAs that contain ester linkages ${ }^{5}$.

In this paper, we investigated the structure and viscoelastic properties of PEAs in solid and molten states to obtain basic information about the unique thermal adhesive properties of PEA with other polymers.

\section{EXPERIMENTAL}

Four PEAs with different compositions were prepared by melt polycondensation of $\mathrm{ADA}, \mathrm{AA}$, and polyetherdiamine (PEDA; $\mathrm{H}_{2} \mathrm{~N}-(\mathrm{PO})_{3}-(\mathrm{TMO})_{9}-(\mathrm{PO})_{3}-\mathrm{NH}_{2}$, PO: propyleneoxide, TMO: tetramethylenoxide) at $230{ }^{\circ} \mathrm{C}$ for $8 \mathrm{~h}$. Table I shows the relative viscosity $\left(\eta_{\mathrm{r}}\right)$, weight average molecular weight $\left(M_{\mathrm{w}}\right)$, the $M_{\mathrm{w}}$ to number average molecular weight $\left(M_{\mathrm{n}}\right)$ ratio $\left(M_{\mathrm{w}} / M_{\mathrm{n}}\right)$, and the composition ratio of ADA/AA/PEDA of the four PEAs. The PEDA compositions in this table indicate the SS contents of the PEAs. The molecular characteristics of commercially available PA12 (grade $3020 \mathrm{U}$, Ube Industries, Ltd.) are shown for comparison. $\eta_{\mathrm{r}}$, $M_{\mathrm{w}}$, and $M_{\mathrm{w}} / M_{\mathrm{n}}$ of the polymerized PEAs were similar. A higher number in the sample name indicates a higher number of SSs, and thus that the sample is softer.

We processed all samples, including PA12, at $200{ }^{\circ} \mathrm{C}$ and rapidly cooled them on dry ice/methanol to prepare films 
Table I Molecular characteristics of polymers used in this study

\begin{tabular}{ccccc}
\hline \multirow{2}{*}{ Sample name } & $\eta_{\mathrm{r}}{ }^{* 1}$ & $M_{\mathrm{w}}{ }^{*} / 10^{4}$ & $M_{\mathrm{w}} / M_{\mathrm{n}}{ }^{* 2}$ & Composition (wt \%) ${ }^{* 3}$ \\
\cline { 5 - 5 } PEA-1 & 1.90 & 5.6 & 2.5 & ADA/AA/PEDA \\
PEA-2 & 1.96 & 6.4 & 2.9 & $72.3 / 2.2 / 25.5$ \\
PEA-3 & 1.81 & 5.7 & 2.8 & $50.7 / 5.1 / 44.2$ \\
PEA-4 & 1.85 & 5.7 & 2.8 & $31.5 / 5.9 / 62.6$ \\
\hline PA12 & 1.80 & 2.8 & 3.7 & - \\
\hline
\end{tabular}

${ }^{* 1} 0.5 \mathrm{~g} / 100 \mathrm{~mL} m$-cresol, $25{ }^{\circ} \mathrm{C} ;{ }^{* 2} \mathrm{SEC}$-LALLS; ${ }^{* 3}{ }^{1} \mathrm{H}-\mathrm{NMR}$

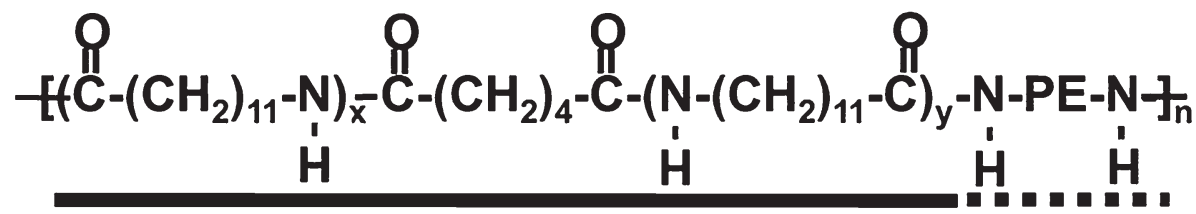

Hard segment

Soft segment

Fig. 1 Chemical structure of PEA. Hard segment consists of 12-aminododecanoic acid and adipic acid. PE stands for poly-ether segment. Details are described in this text.
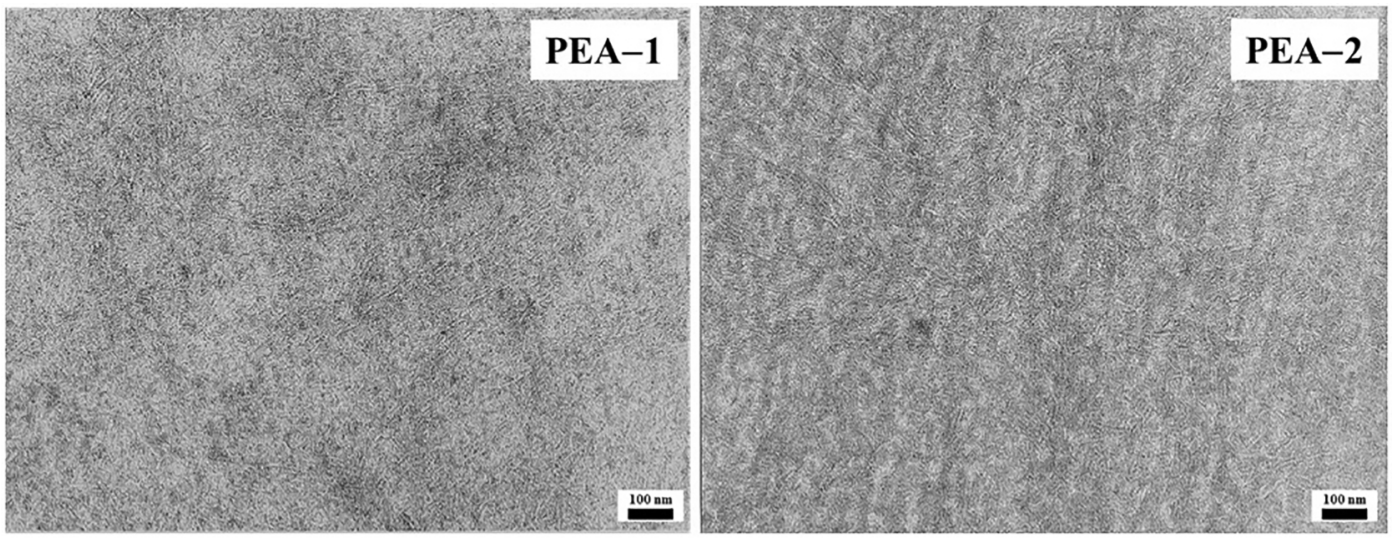

Fig. 2 TEM micrographs of PEA-1 and PEA-2. Stained by $\mathrm{RuO}_{4}$. The magnification is 200,000 .

to suppress structural changes from the molten state accompanying crystallization.

The structure and viscoelastic properties of PEA in the solid state were examined using a transmission electron microscope (JEM-200CX, Japan Electronics Co., Ltd.) and a solid dynamic viscoelastic analyzer (RSA-II, Rheometrics Inc.). The structures of PEAs were observed using ultra-thin films about $100 \mathrm{~nm}$ thick dyed with $\mathrm{RuO}_{4}$. The temperature dependence of the dynamic tensile moduli was measured from -150 to $180{ }^{\circ} \mathrm{C}$. The frequency, applied strain, and heating rate were $10 \mathrm{~Hz}, 0.05 \%$, and $2{ }^{\circ} \mathrm{C} / \mathrm{min}$, respectively. Rectangular samples ( $5 \mathrm{~mm}$ wide, $15 \mathrm{~mm}$ long, 100-150 $\mu \mathrm{m}$ thick) of the four PEAs and PA12 were used. However, we measured the $T_{\mathrm{g}}$ of PEDA as $-78^{\circ} \mathrm{C}$ by using a porous polypropylene membrane impregnated with PEDA because PEDA is a liquid.

The angular frequency dependence of the viscoelastic functions of PEAs in the molten state were measured with a rheometer (ARES, TA Instruments) fitted with a cone and plate with a diameter of $25 \mathrm{~mm}$ and a cone angle of $0.1 \mathrm{rad}$. The angular frequency range and temperature range were 250 to $0.1 \mathrm{rad} / \mathrm{s}^{-1}$ and 200 to $140{ }^{\circ} \mathrm{C}$, respectively.

\section{RESULTS AND DISCUSSION}

The transmission electron microscopy (TEM) images of PEA-1 and PEA-2 are shown in Fig. 2. Although all PEA samples were dyed strongly with $\mathrm{RuO}_{4}$, it was difficult to see the heterogeneously oriented lamella structures about $3 \mathrm{~nm}$ thick and 100-300 nm long. We have previously observed heterogeneous microphase-separated structures several tens of nanometers in size, but not lamella structures, by atomic force microscopy in cross sections of injection-molded PEAs with chemical structures similar to the PEAs in this study ${ }^{6}$. However, we did not observe a heterogeneous microphase-separated structure in our TEM experiments. These 


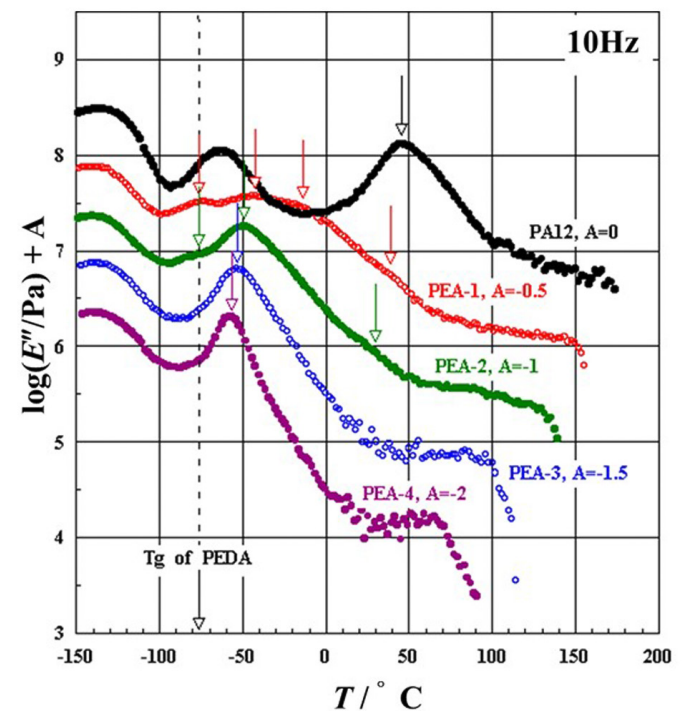

Fig. 3 Temperature dependence of $E^{\prime \prime}$ for four PEA samples and PA12 at $10 \mathrm{~Hz}$.

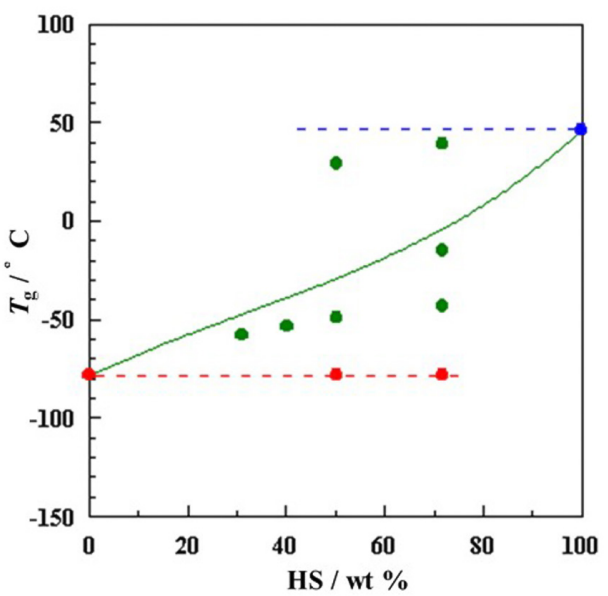

Fig. 4 Dependence of glass transition temperatures upon HS content for four PEA samples.

results suggest that the heterogeneous microphase-separated structure we observed was formed by the injection molding process. We and other researchers have also found that the same types of PEA used in this study have spherulite structures, similar to other PEAs ${ }^{4}$, but these higher-order structures, including the spherulite structures, will be reported separately.

Figure 3 shows the temperature dependence curves of loss elastic modulus $E^{\prime \prime}$ of four PEAs together with that of PA12. For clarity, the $E^{\prime \prime}$ curves of the four PEAs were shifted along the vertical axis. The arrows in Fig. 3 show the relaxation peak positions related to a $T_{\mathrm{g}} \mathrm{S}$ in each polymer. The broken line in the figure represents the $T_{\mathrm{g}}\left(-78^{\circ} \mathrm{C}\right)$ of PEDA, which is the SS in the PEAs.

In the $E^{\prime \prime}$ curve of PA12, three relaxation peaks were observed in addition to a sharp decrease associated with melting around $170{ }^{\circ} \mathrm{C}$. Relaxation peaks around -65 and $-135^{\circ} \mathrm{C}$ were due to the segmental motions of the amide groups and

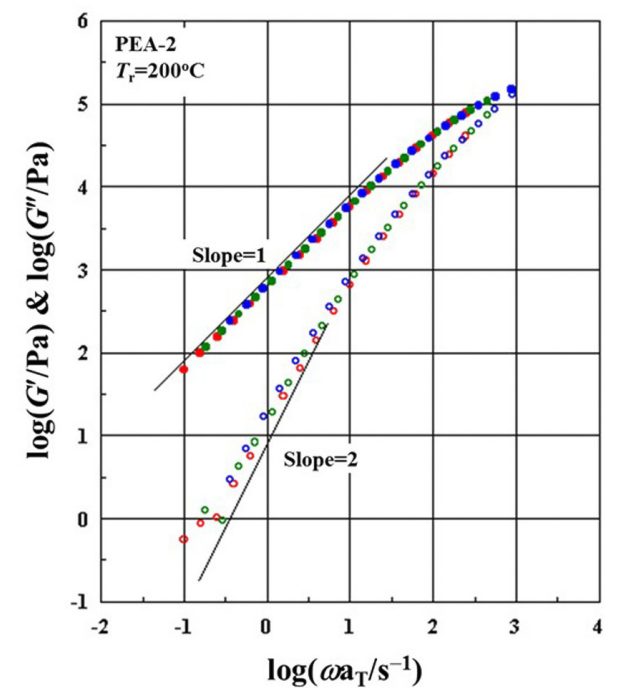

Fig. 5 Master curves of $G^{\prime}$ and $G^{\prime \prime}$ for PEA-2 at $200^{\circ} \mathrm{C}$.

$-\mathrm{CH}_{2}$ - groups between the amide linkages in the PA12 molecular chain, respectively, and a peak for the $T_{\mathrm{g}}$ of PA12 was observed around $47^{\circ} \mathrm{C}^{8)}$. The $E^{\prime \prime}$ curves of PEA-1 and PEA-2 contained four and three glass transition peaks, respectively. In contrast, the $E^{\prime \prime}$ curves of PEA-3 and PEA-4 only contained single glass transition peaks. Figure 4 shows the relationship between $T_{\mathrm{g}} \mathrm{s}$ and HS content for the four PEAs. The solid curve in Fig. 4 indicates $T_{\mathrm{g}}$ calculated by the Fox equation ${ }^{9)}$ from $T_{\mathrm{g}} \mathrm{s}$ of the HS and SS of the PEAs. The main peaks of the four PEAs are near the curve. Furthermore, in the region with a higher HS content, there are two relaxation peaks near the HS and SS glass transition peaks. These results suggest that the amorphous states of PEA-1 and PEA-2 have a typical domain-boundary mixing effect and as the HS content of the PEA decreases, the HS and SS mixed state changes from the domain-boundary mixing to the mixing-in-domain effect. Furthermore, the amorphous states of PEA-3 and PEA-4 show a mixing-in-domain effect ${ }^{10)}$. In other words, the mixing state of the HSs and SSs in the PEAs strongly depends on their compositions, and the HSs and SSs are compatible in the amorphous region when the HS content is $40 \%$ or less.

Figure 5 shows the master curves of storage elastic modulus $G^{\prime}$ and loss elastic modulus $G^{\prime \prime}$ of PEA-2 at the reference temperature of $200{ }^{\circ} \mathrm{C}$. The two straight lines with slopes of 2 and 1 are shown as visual guides in the figure. Almost all of the experimental points for each temperature lie on the master curves of $G^{\prime}$ and $G^{\prime \prime}$ for PEA-2, indicating that PEA-2 is a simple thermodynamic liquid. The master curves were obtained for the other PEAs (data not shown). In the lower-angular-frequency region, the slope of $G^{\prime}$ is smaller than 2 , but this is because the molecular weight distribution 


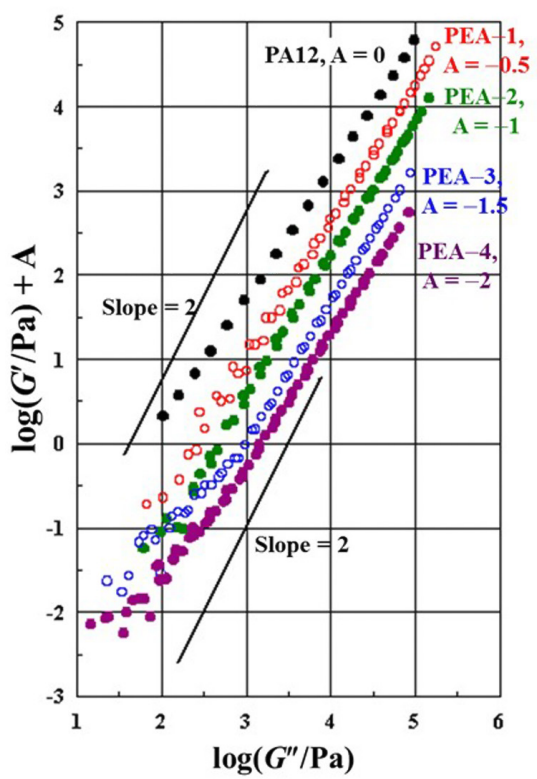

Fig. 6 Relationship between $G^{\prime}$ and $G^{\prime \prime}$ for all polymers.

of PEA-2 is slightly broader. Figure 6 shows the relationship between $G^{\prime}$ and $G^{\prime \prime}$ in the molten state for all the polymers used in this study. For clarity, the $G^{\prime}$ curves of the four PEAs are shifted along the vertical axis. The two straight lines have slopes of 2. For all PEAs, including PA12, the slopes are slightly less than two. In particular, the gradients of PEA-3 and PEA-4 in the low-modulus region appear slightly broader. However, we believe that these behaviors do not arise directly from their heterogeneous structure. The four PEAs in the molten state are general homogeneous liquids like PA12 because we did not observe a long relaxation mechanism, which is a characteristic of a polymer liquid with a heterogeneous structure ${ }^{11,12)}$.

\section{CONCLUSION}

We observed lamella structures in all PEA samples, but no microphase separation structures consisting of domains of several tens of nanometers were formed in the solid state. The HS and SS mixing state strongly depended on the PEA composition. In contrast, the PEAs were uniform liquids in the molten state.

\section{ACKNOWLEDGMENTS}

The authors would like to thank Ube Industries, Ltd. for permission to publish this paper.

\section{REFERENCES}

1) Deleens G, "Thermoplastics Elastomers, A Comprehensive Review", Chapter 9B, Hansen Publisher, 1987.

2) Hatfield GR, Guo Y, Killinger WE, Andrejak RA, Roubicek PM, Macromolecules, 26, 6350 (1993).

3) Ukielski R, Polymer, 41, 1893 (2000).

4) Sheth JP, Xu J, Wilkes GL, Polymer, 44, 743 (2003).

5) Akagawa Y, Plastics, 2005, 44.

6) "Netsukasoseierasutoma- no Zairyousekkei to Seikeikakou", 148, Gijyutsujyouhoukyoukai, 2007.

7) Takemura Y, Nippon Gomu Kyokaishi, 83, 269 (2010).

8) Kawaguchi T, J Appl Polym Sci, 2, 56 (1959).

9) Fox TG, Bull Am Phys Soc, 1, 123 (1956).

10) Hashimoto $\mathrm{T}$, Tsukahara $Y$, Tachi $\mathrm{K}$, Kawai H, Macromolecules, 16, 648 (1983).

11) Graebling D, Muller R, Palierne JF, Macromolecules, 26, 320 (1993).

12) Maeda S, Kamei E, Nihon Reoroji Gakkaishi, 22, 145 (1994). 